\title{
Residual Resistance Data from Cavity Production Projects at Jefferson Lab
}

\author{
Gianluigi Ciovati, Rongli Geng, John Mammosser, and Jeffrey W. Saunders
}

\begin{abstract}
A fundamental limitation towards achieving high quality factors in superconducting radio-frequency cavities is the so-called residual resistance. Understanding and controlling the residual resistance has important implications towards improving the efficiency and reduce the operating cost of continuous wave superconducting linear accelerators. In this contribution we will report on the residual resistance values obtained from measurements of the quality factor of a large set of cavities, with resonant frequency between $805 \mathrm{MHz}$ and $1.5 \mathrm{GHz}$, all of them processed and tested at Jefferson Lab. Surface treatments included both buffered chemical polishing and electropolishing. The results indicate an approximate value of the residual resistance of about 7-10 $\mathrm{n} \Omega$.
\end{abstract}

Index Terms-Niobium, Superconducting accelerator cavities, Superconducting devices.

\section{INTRODUCTION}

$\mathrm{T}$ HE quality factor $\left(Q_{0}\right)$ of radio-frequency (RF) cavities used in particle accelerators is related to the surface resistance $\left(R_{\mathrm{s}}\right)$ of the cavity wall by the following equation:

$$
Q_{0}=\frac{G}{R_{s}} .
$$

$G$ is the so-called geometry factor which depends only on the cavity shape. If superconducting niobium is used to fabricate the cavity, the surface resistance at low field (peak surface magnetic flux density, $B_{\mathrm{p}}$, of about $10-20 \mathrm{mT}$ ) is the sum of the so-called BCS surface resistance $\left(R_{\mathrm{BCS}}\right)$, derived from the Bardeen-Cooper-Schrieffer (BCS) theory of superconductivity, and the residual resistance $\left(R_{\mathrm{res}}\right)$.

A frequently used approximate expression for $R_{B C S}$, valid at temperature $T<T_{\mathrm{C}} / 2$ ( $T_{\mathrm{c}}$ is the critical temperature), is given by [1]

$$
R_{B C S}(T, f)=\frac{A}{T} f^{2} e^{-\Delta / k_{B} T} .
$$

$f$ is the cavity resonant frequency, $\Delta$ is the energy gap at the temperature $T, k_{\mathrm{B}}$ is the Boltzmann constant and $A$ is a fore

Manuscript received 3 August 2010. This manuscript has been authored by Jefferson Science Associates, LLC under U.S. DOE Contract No. DE-AC0506OR23177. The U.S. Government retains a non-exclusive, paid-up, irrevocable, world-wide license to publish or reproduce this manuscript for U.S. Government purposes.

G. Ciovati, R. Geng and J. Mammosser are with the Thomas Jefferson National Accelerator Facility, Newport News, VA 23606 USA (phone: 757269-5945; fax: 757-269-7658; e-mail: gciovati@jlab.org).

J. W. Saunders was with Thomas Jefferson National Accelerator Facility, Newport News, VA 23606 USA. He is now with Oak Ridge National Laboratory, Oak Ridge, TN 37831 USA (e-mail: saundersjw@ornl.gov). factor which depends on material parameters such as the coherence length $(\xi)$, the London penetration depth $\left(\lambda_{L}\right)$ and the electrons mean free path $(l)$. Due to the exponentially decreasing $R_{\mathrm{BCS}}$ at lower temperatures, the $Q_{0}$ would become infinitely high at $0 \mathrm{~K}$ if the only contribution to $R_{\mathrm{s}}$ would be due to thermally activate quasi-particles, as calculated using the BCS theory. Experimentally, it was found that the $\mathrm{Q}_{0}$ is limited at low temperatures by a temperature independent $R_{\text {res }}$ [2]. A brief review of the various possible mechanisms contributing to $R_{\text {res }}$ will be given in the next section. Understanding the origin of $R_{\text {res }}$ and developing processes which will limit its contribution to the $\mathrm{Q}_{0}$ will ultimately reduce the power dissipated by the cavity into the He bath. This will have a significant impact in lowering the cost of modern continuous-wave (CW) superconducting RF (SRF) accelerators used in nuclear physics or in free-electron lasers. Since many phenomena can introduce residual losses, it is difficult to estimate a priori the value of $R_{\text {res }}$ one can expect for a certain cavity frequency and preparation process. Cavity production projects such as for the Spallation Neutron Source (SNS) accelerator, the refurbishment of several cryomodules of the CEBAF accelerator and the R\&D on cavities for the proposed International Linear Collider (ILC) offer the possibility of obtaining statistically significant information on the value of $R_{\text {res }}$ for cavities with different frequency and surface treatment. This article shows the $R_{\text {res }}$ results obtained from 60-100 RF tests of cavities for each of the projects mentioned above.

\section{REVIEW OF CONTRIBUtions TO $\mathrm{R}_{\mathrm{RES}}$}

In this section we will briefly review various mechanisms which may contribute to $R_{\text {res }}$. These are reviewed in greater detail in [3] and references therein. A well-known cause of residual losses is that caused by trapped magnetic flux in the superconductor, either due to Earth's magnetic field or due to thermo-electric currents generated by temperature gradients during the cavity cool-down. The contribution to the residual resistance can be estimated as

$$
R_{\text {res }}=\alpha H_{d c} \sqrt{f} .
$$

$f$ is units of $\mathrm{GHz}, H_{\mathrm{dc}}$ is the residual DC magnetic field and $\alpha$ $=0.2-0.3 \mathrm{n} \Omega / \mathrm{mG}$ for $\mathrm{Nb}$ at $\mathrm{GHz}$ frequencies [2] is a factor which depends on the flux trapping efficiency, on the upper critical field of the superconductor and the normal state resistivity.

Another well-known cause of residual losses is the formation of a normal conducting hydride phase if the bulk hydrogen concentration in $\mathrm{Nb}$ is greater than about $10 \mathrm{wppm}$ 
and if the cavity cool-down rate is slower than about $1 \mathrm{~K} / \mathrm{min}$ in the temperature range 70-150 K. Experiments have shown that $R_{\text {res }}$ values up to about $100 \mathrm{n} \Omega$, depending on the time spent in 70-150 $\mathrm{K}$ temperature region, may result due to hydride formation [2].

Since the surface of a Nb cavity is covered by a 3-5 nm thick oxide layer, additional sources of $R_{\text {res }}$ include dielectric losses and electric interface losses [3]. Dielectric losses due to the natural oxide layer have been estimated to be negligible (< $10^{-11} \mathrm{n} \Omega$ ) because of the low $\tan \delta$ and high dielectric constant of $\mathrm{Nb}_{2} \mathrm{O}_{5}$. Nevertheless, dust particle contamination on the cavity surface may introduce dielectric losses in the nanohm range. Electric interface losses are due to the presence of localized electron states, with density $n_{\mathrm{L}}$, inducing the formation of interface states between the oxide and the metal. These interface states may cause additional losses due to resonant absorption of photons from the RF field. An estimate of these losses yields

$$
R_{r e s} \approx \frac{2 \pi \cdot 10^{-6}}{\varepsilon_{r}^{2}} \Omega \approx 2 \pi \cdot 10^{-9} \Omega
$$

where $\varepsilon_{\mathrm{r}} \sim 30$ is the relative dielectric constant of $\mathrm{Nb}_{2} \mathrm{O}_{5}$.

The interface states also mediate the efficiency in the conversion of RF waves at the surface into phonons, another mechanism which can produce residual losses. An estimate of this contribution yields

$$
R_{\text {res }}<5 \cdot 10^{-7}(f / \mathrm{GHz})^{2} \Omega
$$

The lowest values of $R_{\text {res }}$ which have been measured in $\mathrm{Nb}$ are about $0.5-2 \mathrm{n} \Omega$ [4] but these are difficult to reproduce regularly.

\section{EXPERIMENTAL RESULTS}

The best method to determine the value of $R_{\text {res }}$ is to measure $Q_{0}$ at low field as a function of temperature, calculate $R_{\mathrm{s}}$ using (1) and fit the data according to the following

$$
R_{s}(T)=R_{B C S}(T)+R_{\text {res }} \text {. }
$$

$R_{\mathrm{BCS}}(T)$ is calculated using the full BCS theory or using the approximated formula given by (2). $Q_{0}$ should be measured down to a temperature where a clear deviation from the exponential dependence shown in (2) is observed.

As the measurement of $Q_{0}(T)$ between $4.3 \mathrm{~K}$ and $1.6 \mathrm{~K}$ is time consuming, it is typically not done during RF tests of cavity production projects, where a measurement at fixed He bath temperature of $Q_{0}$ as a function of the accelerating gradient is sufficient to qualify a cavity for installation in a cryomodule. Therefore we extract the value of $R_{\text {res }}$ using (6) for a single temperature data point. The value of $R_{\mathrm{BCS}}(T)$ is calculated from the full BCS theory using the computer code developed by Halbritter [5] with the following material parameters, which well describe $\mathrm{Nb}$ after the typical cavity surface preparation process [6]: $\Delta / k_{\mathrm{B}} T_{\mathrm{C}}=1.82, \lambda_{\mathrm{L}}=32 \mathrm{~nm}, \xi=$ $39 \mathrm{~nm}, T_{\mathrm{C}}=9.25 \mathrm{~K}$ and $l=200 \mathrm{~nm}$. The $R_{\mathrm{BCs}}$-value for diffuse, rather than specular, electron surface reflection is used. The typical measurement error of $Q_{0}$ is $10-15 \%$ whereas given $\Delta / k_{\mathrm{B}} T_{\mathrm{c}}$ ranging between 1.81 and 1.83 , and $l$ ranging between 100 and $300 \mathrm{~nm}$, we estimate the uncertainty on the value of $R_{\mathrm{BCS}}$ to be about $15 \%$. These will be systematic uncertainties in the determination of $R_{\text {res }}$.

If the cavities are baked at low-temperature $\left(120{ }^{\circ} \mathrm{C}\right.$ for 48 h) before the RF test, $R_{\mathrm{BCS}}$ is reduced by about $50 \%$ than prior to baking [6]. This is described by an increase of the energy gap value and a reduction of the mean free path, so that the values used to calculate $R_{\mathrm{BCS}}$ when this treatment is applied are [6]: $\Delta / k_{\mathrm{B}} T_{\mathrm{C}}=1.89$ and $l=20 \mathrm{~nm}$. The uncertainty in the estimation of $R_{\mathrm{BCS}}$ is also about $15 \%$ in this case.

All the cavities are made of high purity (residual resistivity ratio $>250$ ) niobium.

\section{A. SNS Cavities}

Thirty-eight medium- $\beta$ (MB) and 56 high- $\beta$ (HB) 805 MHz, six-cell cavities were processed and tested at Jefferson Lab between 2002 and 2004 for the SNS project. Details about the cavity shape and electromagnetic parameters are given in [7], [8]. The main processing steps after fabrication consisted of bulk buffered chemical polishing (BCP), removing about 120 $\mu \mathrm{m}$ from the cavity inner surface, followed by heat treatment in a ultra-high vacuum (UHV) furnace at $600^{\circ} \mathrm{C}$ for $10 \mathrm{~h}$, final $20 \mu \mathrm{m}$ removal by BCP and high-pressure rinsing (HPR) with ultra-pure water. The cavities were tested at $2.1 \mathrm{~K}$. The value of $R_{\mathrm{BCS}}$ at $805 \mathrm{MHz}$ and $2.1 \mathrm{~K}$ is $7.2 \mathrm{n} \Omega$ and $4.2 \mathrm{n} \Omega$, before and after baking, respectively. The values of $R_{\text {res }}$, obtained subtracting $R_{\mathrm{BCS}}$ from $R_{\mathrm{s}}$ calculated from the measured value of $Q_{0}(2.1 \mathrm{~K})$ at a $B_{\mathrm{p}}$-value of $18 \mathrm{mT}$ using (1), are shown in Figs. 1 and 2 for the $\mathrm{MB}$ and $\mathrm{HB}$ cavities, respectively.

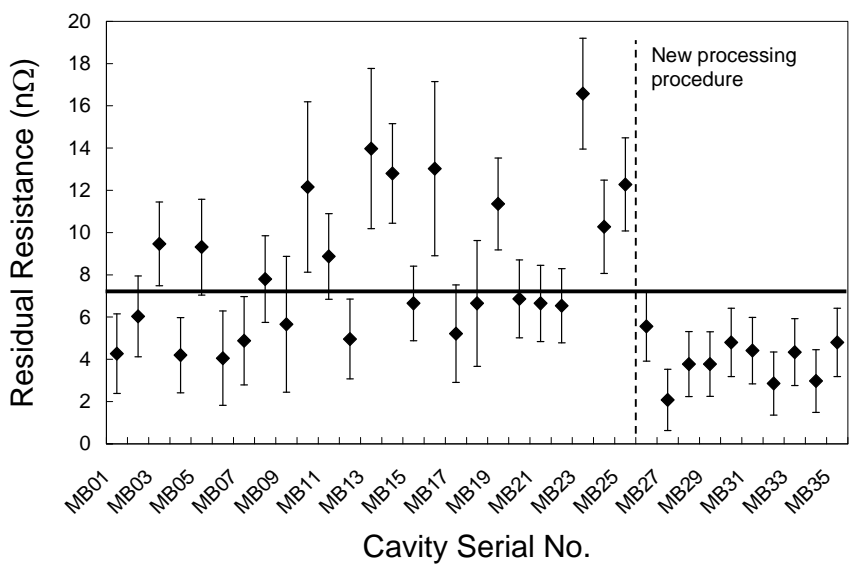

Fig. 1. Residual resistance data calculated from the low-field $Q_{0}$ measured at $2.1 \mathrm{~K}$ for the $805 \mathrm{MHz}$ SNS-MB cavities. The solid line corresponds to the average value of $R_{\text {res. }}$ None of the cavities were baked at $120^{\circ} \mathrm{C}$. The position of the cavity serial No. on the horizontal axis corresponds to the date the cavity was tested.

The average value of $R_{\text {res }}$ for the SNS-MB cavities is $7.1 \pm$ $2.2 \mathrm{n} \Omega$. Since the high-power RF tests of the SNS-MB cavities showed strong field emission limiting the attainable accelerating gradient, improvements in the cleaning procedures were made starting with cavity MB26. The main changes were the following:

1) The amount of material removal after heat treatment at $600{ }^{\circ} \mathrm{C}$ increased from about $10 \mu \mathrm{m}$ to about $20 \mu \mathrm{m}$.

2) The amount of rinsing immediately following BCP was increased to ten fill-and-dump cycles with ultra-pure water.

3) Two 2 h long HPR cycles were done instead of just one 
cycle.

These changes not only reduced the incidence of field emission during high-power RF test but also resulted in higher Q-values. The average value of $R_{\text {res }}$ before the changes were implemented was $8.4 \pm 2.4 \mathrm{n} \Omega$ which decreased to $3.9 \pm 1.6$ $\mathrm{n} \Omega$ after the new procedures were adopted. The average value of $R_{\text {res }}$ for the SNS-HB cavities which were not baked at 120 ${ }^{\circ} \mathrm{C}$ is $6.3 \pm 1.9 \mathrm{n} \Omega$ whereas it is $8.1 \pm 1.8 \mathrm{n} \Omega$ for baked cavities.

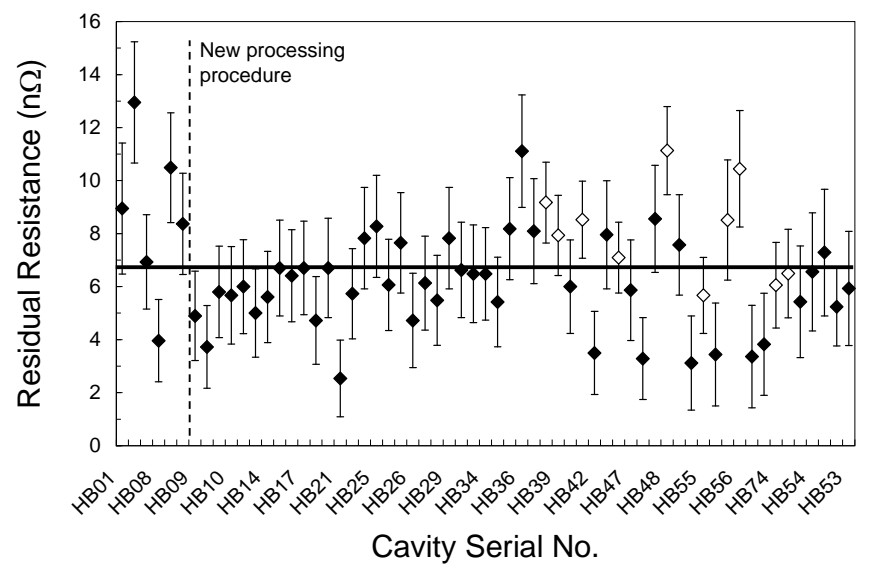

Fig. 2. Residual resistance data calculated from the low-field $Q_{0}$ measured at $2.1 \mathrm{~K}$ for the $805 \mathrm{MHz}$ SNS-HB cavities. The solid line corresponds to the average value of $R_{\text {res }}$. The empty symbols are for cavities that were baked at $120^{\circ} \mathrm{C}$ for $48 \mathrm{~h}$. The position of the cavity serial No. on the horizontal axis corresponds to the date the cavity was tested.

A histogram plot of the $R_{\text {res }}$ values for all SNS cavities which had not been baked is shown in Fig. 3. The data follow a Gaussian distribution with a fit correlation factor $\left(r^{2}\right)$ of 0.87 . The average value of $R_{\text {res }}$ for the SNS cavities is $6.6 \pm$ 0.3 (stat) \pm 2.0 (sys) $\mathrm{n} \Omega$.

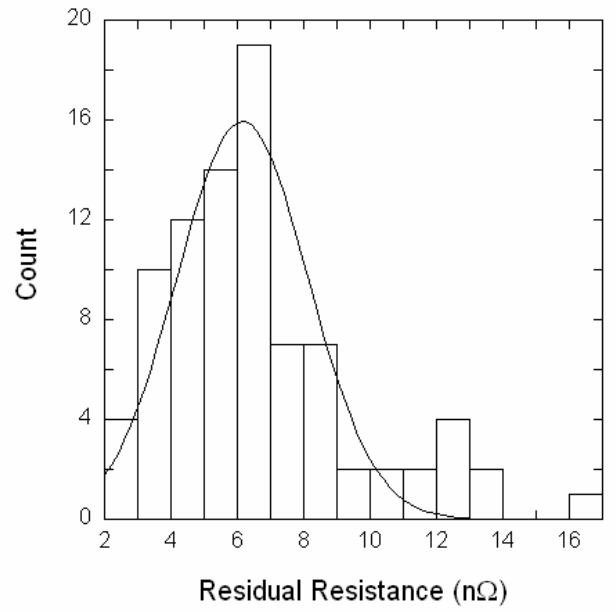

Fig. 3. Histogram plot of $R_{\text {res }}$ for the $805 \mathrm{MHz}$ SNS cavities. The solid line is a fit with a Gaussian distribution.

\section{B. CEBAF Cavities}

Approximately 80 5-cell, $1497 \mathrm{MHz}$ cavities which were installed in the lowest performing cryomodules in the CEBAF accelerator at JLab were reworked between 2006 and 2009. Details about the cavity shape and electromagnetic parameters are given in [9]. The cavities were re-processed with the same techniques used for the SNS cavity production and tested for their performance in terms of $Q_{0}$ as a function of accelerating gradient at a He bath temperature of $2.0 \mathrm{~K}$. The calculated value of $R_{\mathrm{BCS}}$ at $1497 \mathrm{MHz}$ and $2.00 \mathrm{~K}$ is $14.9 \mathrm{n} \Omega$. None of the cavities were baked at $120{ }^{\circ} \mathrm{C}$. The values of $R_{\text {res }}$ obtained as described earlier from $Q_{0}(2.0 \mathrm{~K})$ at $B_{\mathrm{p}}=9 \mathrm{mT}$ are shown in a histogram plot in Fig. 4. Some of the cavities were tested multiple times. The data follow a Gaussian distribution with $r^{2}$ $=0.69$. The average value of $R_{\text {res }}$ for the CEBAF cavities is $10.3 \pm 0.4$ (stat) \pm 4.2 (sys) $\mathrm{n} \Omega$.

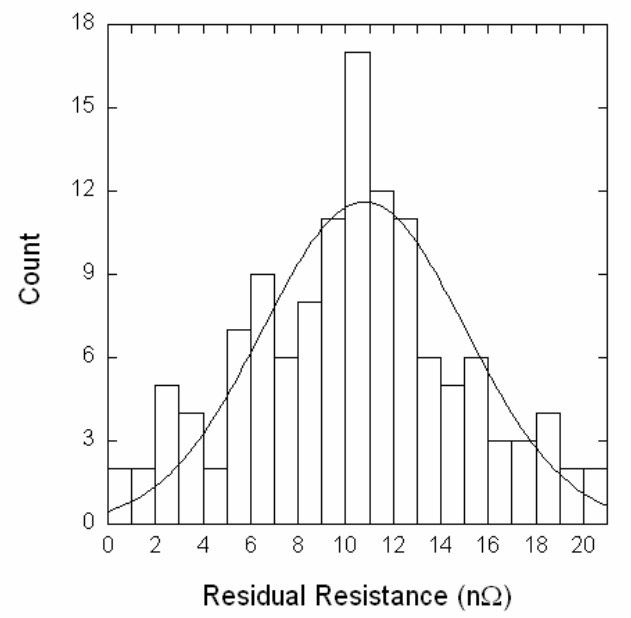

Fig. 4. Histogram plot of $R_{\text {res }}$ for the $1497 \mathrm{MHz}$ CEBAF cavities. The solid line is a fit with a Gaussian distribution.

\section{ILC Cavities}

Twenty-four 9-cell, $1300 \mathrm{MHz}$ cavities for the ILC project have been processed and tested multiple times at JLab since 2007. Details about the cavity shape and electromagnetic parameters are given in [10]. For this project, the method to remove material from the inner cavity surface after fabrication and high-temperature heat-treatment is electropolishing (EP), rather than BCP. In addition, the cavities are routinely baked at $120{ }^{\circ} \mathrm{C}$ for $48 \mathrm{~h}$ prior to $\mathrm{RF}$ testing at $2.0 \mathrm{~K}$. These processes allow achieving peak surface magnetic fields in excess of $140 \mathrm{mT}$ [11].

The calculated value of $R_{\mathrm{BCS}}$ at $1300 \mathrm{MHz}$ and $2.0 \mathrm{~K}$ is 11.6 $\mathrm{n} \Omega$ and $6.7 \mathrm{n} \Omega$ before and after baking, respectively. The values of $R_{\text {res }}$ obtained as described earlier from the $Q_{0}(2.0 \mathrm{~K})$ at $B_{\mathrm{p}}=13-20 \mathrm{mT}$ are shown in Fig. 5. A histogram plot of the $R_{\text {res }}$ values for all ILC cavities which had been baked is shown in Fig. 6. The data follow a Gaussian distribution with a fit with $r^{2}=0.53$. The average value of $R_{\text {res }}$ for the ILC cavities is $8.0 \pm 0.4$ (stat) \pm 2.6 (sys) $\mathrm{n} \Omega$.

\section{DISCUSSION}

The average values of $R_{\text {res }}$ as a function of the cavity resonant frequency are shown in Fig. 7. Although the large systematic uncertainties do not allow us to draw any strong conclusion from the data, they are well fitted $\left(r^{2}=0.90\right)$ with the following dependence:

$$
R_{\text {res }}=a+b f^{2}
$$

where $a=5.3 \mathrm{n} \Omega$ is a frequency-independent term and $b=$ $1.89 \mathrm{n} \Omega / \mathrm{GHz}$.

The values of the mean, the standard deviation and the 
median from the distribution of the $R_{\text {res }}$ data at $805 \mathrm{MHz}, 1300$ $\mathrm{MHz}$ and $1497 \mathrm{MHz}$ are given in Table 1.

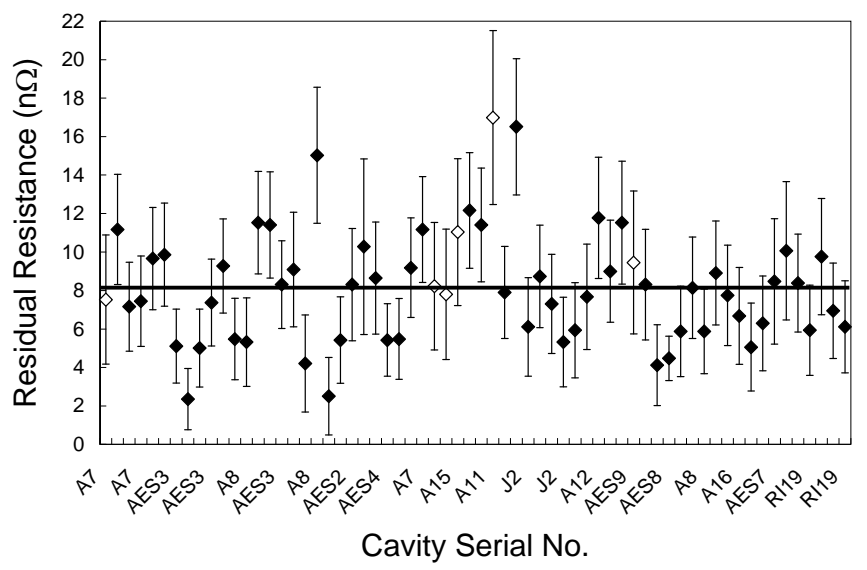

Fig. 5. Residual resistance data calculated from the low-field $Q_{0}$ measured at $2.0 \mathrm{~K}$ for the $1300 \mathrm{MHz}$ ILC cavities. The solid horizontal line corresponds to the average value of $R_{\mathrm{res}}$. The empty symbols are for cavities that were not baked. The position of the cavity serial No. on the horizontal axis corresponds to the date the cavity was tested.

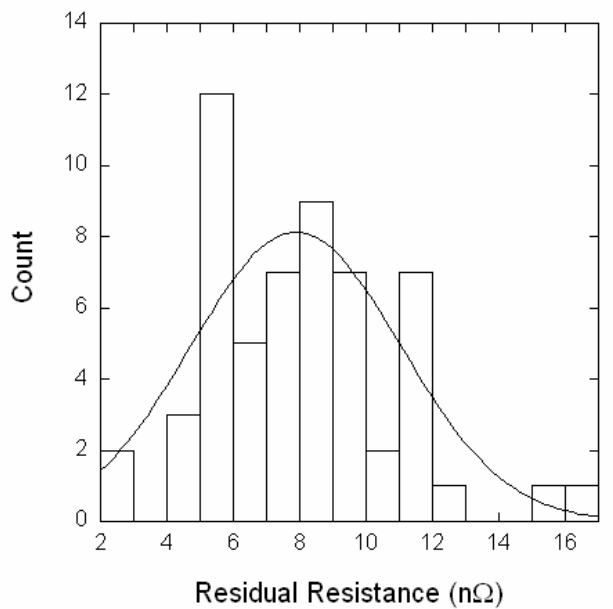

Fig. 6. Histogram plot of $R_{\text {res }}$ for the $1300 \mathrm{MHz}$ ILC cavities. The solid line is a fit with a Gaussian distribution.

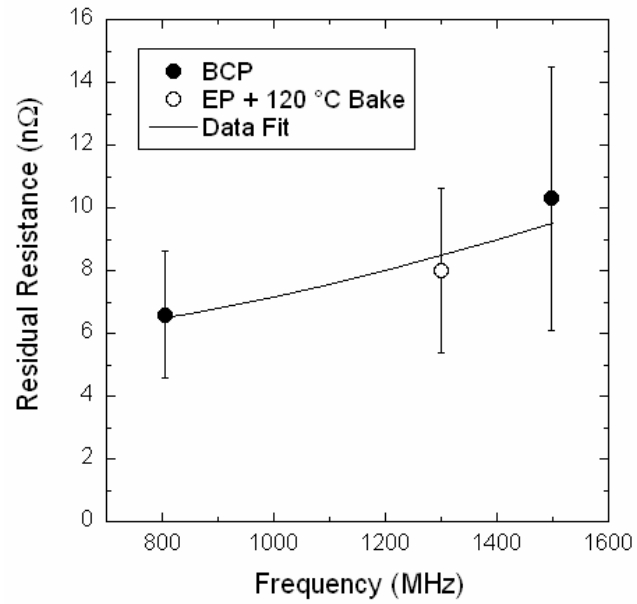

Fig. 7. Average value of $R_{\text {res }}$ as a function of frequency. The data are fitted with (7).

The Earth's magnetic field is shielded inside the vertical cryostats at JLab by a combination of $\mu$-metal sheet and compensation coils. The residual field inside the cryostats in which all the cavities were tested is about $6 \mathrm{mG}$, contributing
1-2 $\mathrm{n} \Omega$ to the residual resistance.

Earlier studies done in the 1970s [12] on a multi-mode cavity showed $R_{\mathrm{res}}$-values greater than about $30 \mathrm{n} \Omega$ at $1.7 \mathrm{GHz}$ and increasing quadratically with frequency. Similar frequency dependence and $R_{\mathrm{res}}$-values greater than about 50 $\mathrm{n} \Omega$ were reported on lead-pleated copper cavities [13]. A model based on the generation of phonons in fissured surfaces by locally excited RF fields was proposed to explain the observed losses and their frequency dependence [13].

A collection of the lowest values of $R_{\text {res }}$ measured in $\mathrm{Nb}$ cavities of different frequencies showed $R_{\mathrm{res}}$-values of about 2 $\mathrm{n} \Omega$ with no significant frequency dependence between 500 $\mathrm{MHz}$ and $20 \mathrm{GHz}$ [14]. The results reported in this article are consistent with the presence of a frequency-independent contribution to the residual losses of about $5 \mathrm{n} \Omega$ added to a $f^{2}$ dependent term. There is no significant dependence of $R_{\text {res }}$ on the surface preparation method, BCP or EP followed by baking.

\begin{tabular}{|c|c|c|c|}
\hline & 805 MHz & $1300 \mathrm{MHz}$ & $1497 \mathrm{MHz}$ \\
\hline Mean & $6.6 \pm 2.0 \mathrm{n} \Omega$ & $8.0 \pm 2.6 \mathrm{n} \Omega$ & $10.3 \pm 4.2 \mathrm{n} \Omega$ \\
\hline $\begin{array}{l}\text { Standard } \\
\text { deviation }\end{array}$ & $2.8 \mathrm{n} \Omega$ & $2.8 \mathrm{n} \Omega$ & $4.6 \mathrm{n} \Omega$ \\
\hline Median & $6.1 \mathrm{n} \Omega$ & $7.9 \mathrm{n} \Omega$ & $10.2 \mathrm{n} \Omega$ \\
\hline $\begin{array}{l}\text { No. of } \\
\text { cavities }\end{array}$ & 83 & 24 & 83 \\
\hline No. of tests & 86 & 57 & 127 \\
\hline
\end{tabular}

\section{CONCLUSION}

Large-scale SRF cavity production projects provide an opportunity to study the behavior of RF residual losses in a statistically significant way. The data from such projects carried out at JLab over the past 8 years on cavities ranging from $805 \mathrm{MHz}$ to $1497 \mathrm{MHz}$ indicate an average value of $R_{\text {res }}$ of about 7-10 n $\Omega$. The uncertainty in the average $R_{\text {res }}$-values is 2-4 $\mathrm{n} \Omega$, mostly systematic. No significant dependence of $R_{\text {res }}$ on surface treatment was found. On the frequency dependence of $R_{\text {res }}$, the data can be described by the sum of a frequencyindependent term and an $f^{2}$-dependent term, in agreement with theoretical models.

\section{REFERENCES}

[1] H. Padamsee, J. Knobloch, and T. Hays, RF Superconductivity for Accelerators. New York, NY: Wiley \& Sons, 1998, p.88.

[2] H. Padamsee, J. Knobloch, and T. Hays, RF Superconductivity for Accelerators. New York, NY: Wiley \& Sons, 1998, pp. 171-177.

[3] J. Halbritter, "On RF residual losses in superconducting cavities," in Proc. 2nd Workshop on RF Supercond., Geneva, Switzerland, 1984, pp. 427-446.

[4] A. Septier and Nguyen Tuong Viet, "Microwave applications of superconducting materials," J. Phys. E: Sci. Instrum., vol. 10, no. 12, pp. 1193-1207, Dec. 1977.

[5] J. Halbritter, "FORTRAN-Program for the computation of the surface impedance of superconductors," Karlsruhe Nuclear Research Center, Technical Note 3/70-6, June 1970. 
[6] G. Ciovati, "Effect of low temperature baking on the radio-frequency properties of niobium superconducting cavities for particle accelerators," J. Appl. Phys., vol. 96, no. 3, pp. 1591-1600, Aug. 2004.

[7] D. Barni, A. Bosotti, G. Ciovati, C. Pagani, and P. Pierini, "SC cavity design for the $700 \mathrm{MHz}$ TRASCO linac,” Proc. of the 2000 European Particle Accelerator Conf., Vienna, Austria, 2000, pp. 2019-2021.

[8] J. P. Ozelis, J. R. Delayen, and J. Mammosser, "Performance overview of the superconducting RF cavities for the Spallation Neutron Source linac," Proc. of the 2005 Particle Accelerator Conf., Knoxville, 2005, pp. 4048-4050.

[9] R. M. Sundelin, "High gradient superconducting cavities for storage rings,” IEEE Trans. Nucl. Sci., vol. 32, no. 5, pp. 3570-3573, Oct. 1985.

[10] B. Aune et al., "Superconducting TESLA cavities," Phys. Rev. ST Accel. Beams, vol. 3, no. 9, 092001, Sept. 2000.
[11] L. Lilje, “Achievement of $35 \mathrm{MV} / \mathrm{m}$ in the TESLA superconducting cavities using electropolishing as a surface treatment," Proc. of the 2004 European Particle Accelerator Conf., Lucerne, Switzerland, 2004, pp. 129-131.

[12] W. Bauer, S. Giordano, and H. Hahn, "Measured frequency dependence of the surface resistance of superconducting niobium," $J$. Appl. Phys., vol. 45, no. 11, pp. 5023-5026, Nov. 1974,

[13] J. Halbritter, “Surface residual resistance of high-Q-superconducting resonators,” J. Appl. Phys., vol. 42, no. 1, pp. 82-87, Jan. 1971.

[14] H. Padamsee, "The science and technology of superconducting cavities for accelerators,” Supercond. Sci. Technol., vol. 14, no. 4 pp. R28-R51, Apr. 2001. 